\title{
Measurement and Imaging of Planar Electromagnetic Phantoms Based on NMR Imaging Methods
}

\author{
I. Frollo, P. Andris, J. Přibil, L. Vojtíšek, T. Dermek, L. Valkovič \\ Institute of Measurement Science, SAS, Dúbravská 9, 84104 Bratislava, Slovakia, frollo@savba.sk
}

\begin{abstract}
Planar electromagnetic phantom design for measurement and imaging using NMR has been performed. Electromagnetic phantom computation and testing on a NMR 0.178 Tesla Esaote Opera imager were accomplished. The classical geometrical and chemical phantoms are generally used for testing of NMR imaging systems. They are simple cylindrical or rectangular objects with different dimensions and shapes with holes filled with specially prepared water solutions. In our experiments a homogeneous phantom (reference medium) - a container filled with water - was used. The resultant image represents the magnetic field distribution in the homogeneous phantom. An image acquired by this method is actually a projection of the sample properties onto the homogeneous phantom. The goal of the paper is to map and image the magnetic field deformation using NMR imaging methods. We are using a double slender rectangular vessel with constant thickness filled with specially prepared water solution in our experiments. For detection a carefully tailored gradient-echo imaging method, susceptible to magnetic field homogeneity, was used.
\end{abstract}

Keywords: Magnetic measurement, magnetic phantom, magnetic resonance imaging, quality testing

\section{INTRODUCTION}

$\mathrm{T}$ HE NUCLEAR Magnetic Resonance (NMR) measurement and imaging of proton density of biological structures needs to create basic physical conditions, stationary, gradient and radio-frequency (RF) magnetic fields. A biological or physical object placed into the centre of a measurement region is absorbing the energy during the RF excitation pulse generated by a coil supplied by an external source - RF transmitter. After absorption is finished, the object radiates the energy back to the surrounding space. This energy is detected by an RF receiving coil. The signal is amplified, demodulated and after A/D conversion it submits data. Data accumulated in the buffer of a computer are processed by Fast Fourier Transform and organized in a matrix. The matrix represents a spatial distribution of the measured parameters (proton density, relaxation times, etc.).

It is not possible to use standard MRI methods for imaging of objects that do not incorporate enough hydrogen atoms and do not generate any NMR signal. Inserting such an object into a stationary homogeneous magnetic field results in field deformation which is proportional to the susceptibility of the sample. If the space in the vicinity of the sample is filled with a water-containing substance, we are able to image this sample. In the case of a homogeneous phantom (e.g. a slender rectangular container filled with water), the acquired image represents a modulation of the local magnetic field, representing the magnetic susceptibility distribution in the sample.

In general, phantoms are artificial objects of known dimensions used for test runs in NMR systems - system tuning and evaluation of image properties. Using phantoms we are able to evaluate image quality, $\mathrm{S} / \mathrm{N}$ ratio measurements, geometrical distortions of images, time stability of the imaging system, ghosts, interferences, and Electromagnetic compatibility (EMC) parameters.

For imaging based on NMR, passive - chemical phantoms are generally used. One can use uniform phantoms: planar, spherical, and cylindrical with a number of geometrical reference objects.

The routine phantoms are made from Plexiglas filled with aqueous solutions e.g. $\mathrm{NiCl}_{2}$ and $\mathrm{NaCl}$.

Advantages of chemical passive phantoms: robustness, handy, stable geometry.

Disadvantages: chemical instability, necessity of refilling the aqueous solution, bubbles on top of the phantom and in the hidden areas, impossible to change the sensitivity of imaging, impossible to use it as a standard for comparison of NMR imagers equipped with different magnetic fields, questionable repeatability.

The goal of the research is to design and verify several types of planar electromagnetic phantoms supplied by small stabilized DC current.

Using an appropriate mathematical model it is possible to calculate the magnetic field generated by the phantoms, but in a real NMR imaging only selected magnetic field distribution components are imaged.

The first attempt of a direct measurement of the magnetic field created by a simple wire fed by a current was reported in [1]. This report describes an experiment in which magnetic fields produced by a small current applied to the forearm of a living subject have been detected in the tissue. Initial experiments were performed in vitro using a cylindrical phantom. The phase-image computed from the real and imaginary images demonstrated the presence of a magnetic field in the cylinder.

A method utilizing the divergence in gradient strength that occurs in the vicinity of a thin current-carrying copper wire was introduced in [2]. Using pulsed gradient spin-echo NMR sequence and a solution of polyethylene oxide in water, in vitro micro images of a sample were presented. The paper demonstrated the effect of molecular diffusion in the vicinity of a thin wire subjected to current pulses. This effect was demonstrated in measurements on polymer solutions and liquid crystals.

A simple experiment with thin, pulsed electrical current 
carrying wire and imaging of a magnetic field using a plastic sphere filled with agarose gel as phantom was published in [3]. Images of the phantom were obtained with and without application of electric current to a straight wire using a spinecho NMR sequence. Because the method proposed in this study does not use phase images, phase unwrapping was not required. The method is designed for the detection of electric currents in biological tissues by observations of surface potentials or surrounding magnetic fields.

Nuclear magnetic resonance imaging is an effective method for indirect mapping of magnetic field distribution. Based on these principles we propose electromagnetic phantoms as an additional tool for measurement and imaging quality testing using NMR imaging methods. Numerical analyses of the magnetic field for arbitrary magnetic susceptibility mapping were described in [4], [5], [6], [7]. Phantom design method for high-field MRI human and systems multilayered spherical head phantom excited by MRI coils were described in [8], [9].

In the first attempts, for testing and calibration, a rectangular flat coil-meander fed by electric current and generating a weak magnetic field was used.

Our task is to map and image the magnetic field deformation using NMR imaging methods. In our experiments we are using slender rectangular vessels with constant thickness filled with specially prepared water. A carefully tailored gradient-echo NMR measuring sequence was used.

\section{SubJeCt \& METHODS}

From the theoretical point of view, we suppose that the conductor position is on the $\mathrm{x}$-axis in the rectangular complex coordinate system (ix, jy, $\mathbf{k z})$ and the diameter of the conductor is neglected.

According to Fig.1, we suppose one conductor limited by two points $(\mathrm{P}, \mathrm{Q})$, lengths of $2 \mathrm{~L}$, with the left - right symmetry. Conductor is fed by currents $+\mathrm{I}$. The basic magnetic field $\mathrm{B}_{0}$ of the NMR imager is parallel with the $\mathrm{z}$ axis. The task is to calculate the $\mathrm{B}_{\mathrm{z}}(\mathrm{x}, \mathrm{y}, \mathrm{z})$ component of the magnetic field near the conductor.

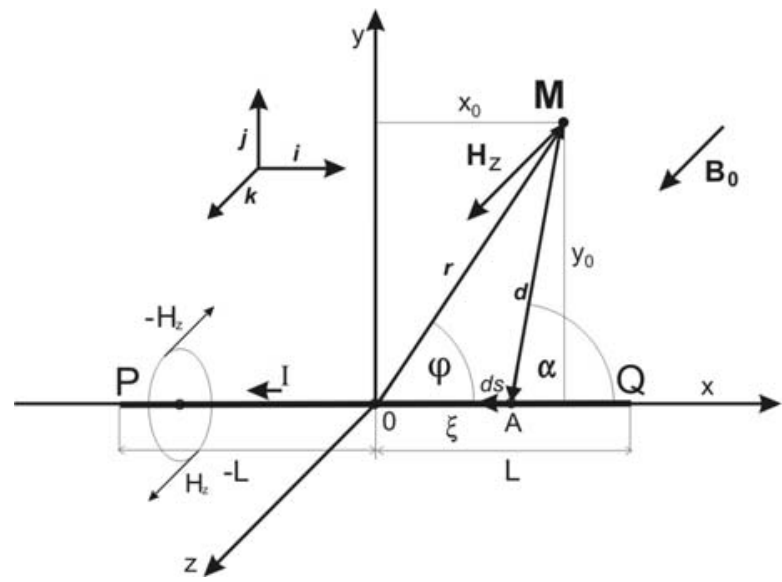

Fig.1. Basic configuration of the limited length conductor PQ placed into the $\mathrm{x}-$ axis.
The formula for the magnetic field in point $\mathrm{M}\left(\mathrm{x}_{0}, \mathrm{y}_{0}\right)$ is supposes that $\mathrm{z}_{0}=0$. For computation of the magnetic field the Biot-Savart law in vector form was used [10].

$$
\mathbf{H}=\frac{\mathrm{I}}{4 \pi} \oint \frac{\mathrm{d} \mathbf{s} \times \mathbf{d}}{\mathrm{d}^{3}}
$$

From the physical interpretation the magnetic field is linesymmetry in compliance with $\mathrm{x}$-axis, and symmetric according to the $\mathrm{yz}-$ plane. The calculation is limited to the first quadrant of the xy - plane. Following the Fig.1 and formula (1) we can write:

$$
\mathrm{d} \mathbf{s} \times \mathbf{d}=\mathrm{ds} \mathrm{d} \sin (\alpha)=\mathrm{d} \xi \mathrm{d} \sin (\alpha)
$$

Using formula (1) we get the basic expression as follows

$$
\mathbf{H}=\mathbf{k} \frac{\mathrm{I}}{4 \pi} \int_{-\mathrm{L}}^{\mathrm{L}} \frac{\sin \alpha}{\mathrm{d}^{2}} \mathrm{~d} \xi
$$

Considering Fig.1 : $\quad \sin \alpha=\frac{\mathrm{r}}{\mathrm{d}} \sin \varphi$,

$\mathrm{d}^{2}=\mathrm{r}^{2}+\xi^{2}-2 \mathrm{r} \xi \cos \varphi, \quad \mathrm{r} \sin \varphi=\mathrm{y}, \quad \mathrm{r} \cos \varphi=\mathrm{x}$

In the complex coordinate system (ix, jy, kz) we get the formula for magnetic field $\mathbf{H}$ in the integral form

$$
\mathbf{H}=\mathbf{k} \frac{\operatorname{Iy}}{4 \pi} \int_{-L}^{\mathrm{L}} \frac{\mathrm{d} \xi}{\sqrt{\left(\mathrm{x}^{2}+\mathrm{y}^{2}+\xi^{2}-2 \xi \mathrm{x}\right)^{3}}} .
$$

After integration of (4) we get the final general formula for magnetic field calculation

$$
H_{z}=\frac{I}{4 \pi y}\left[\frac{L-x}{\sqrt{\left(x^{2}+y^{2}+L^{2}-2 L x\right)^{3}}}+\frac{L+x}{\sqrt{\left(x^{2}+y^{2}+L^{2}+2 L x\right)^{3}}}\right] \text {. (5) }
$$

As a mathematical and physical model a flat meander rectangular coil was designed and a magnetic field using formula (5) for every wire was calculated. The resultant plots of magnetic field relative values are depicted in Fig.2a, and Fig.2b.

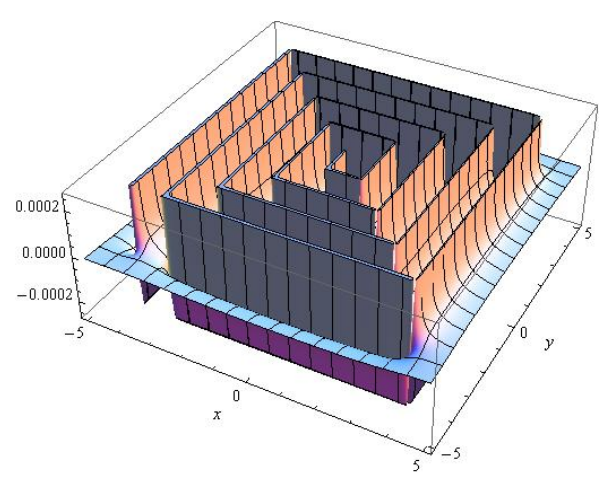

Fig.2a. Calculated magnetic field $\mathrm{H}_{\mathrm{z}}(\mathrm{x}, \mathrm{y})$ of the planar meander rectangular coil, limited plot-range, $3 \mathrm{D}$-plot of relative values. 


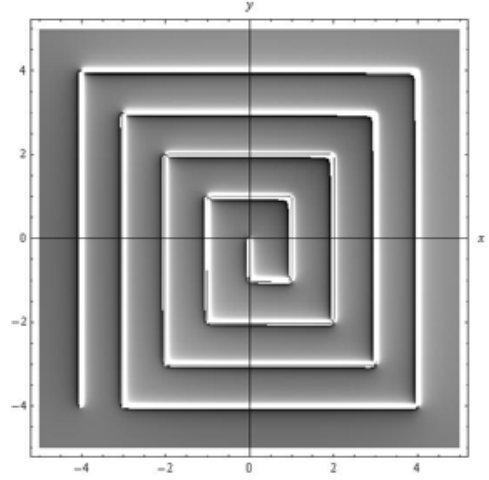

Fig.2b. Calculated magnetic field $\mathrm{H}_{\mathrm{z}}(\mathrm{x}, \mathrm{y})$ of the planar meander rectangular coil. Density-plot of relative values - similar to NMR image.

For a second theoretical example, in the next analysis we suppose two settings:

1. All conductors are creating a rectangle parallel with $y$ axis in the rectangular coordinate system $(\mathrm{x}, \mathrm{y}, \mathrm{z})$.

2. The diameter of conductors and the influence of supply wires are neglected.

According to Fig.3, we assume four conductors creating a rectangle defined by four points $(\mathrm{P}, \mathrm{Q}, \mathrm{R}, \mathrm{T})$ with the left - right symmetry. Conductors are fed by currents $+\mathrm{I}$, -I. The static magnetic field $\mathbf{B}_{0}$ of the NMR imager is parallel with the z-axis.

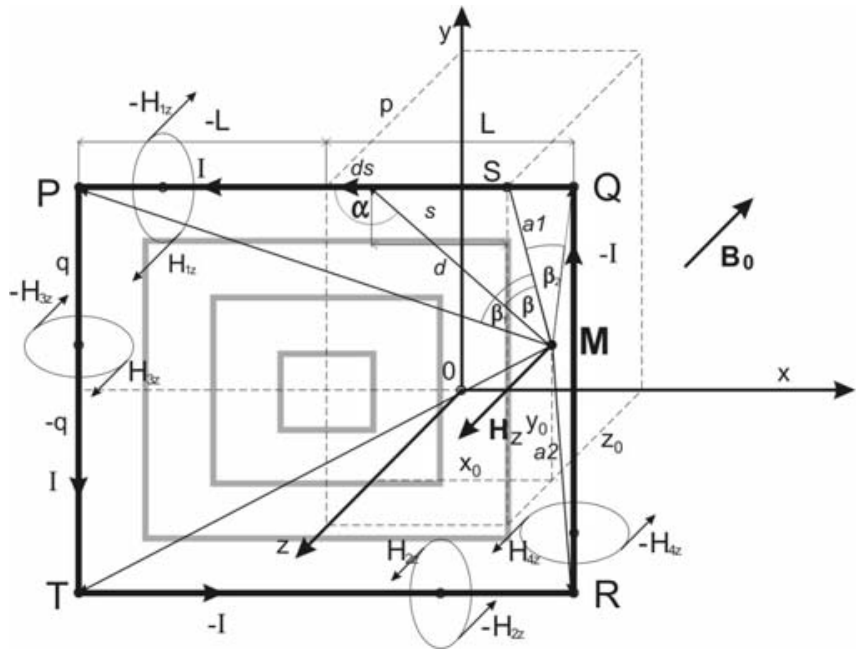

Fig.3. Basic configuration of the four conductor rectangular coil. Three additional rectangular coils are depicted in grey colour.

Let us shortly explain the way of computation. For simplicity we consider only one horizontal conductor fed by currents $+\mathrm{I}$.

Let us derive a formula for magnetic field in point $\mathrm{M}\left(\mathrm{x}_{0}, \mathrm{y}_{0}, \mathrm{z}_{0}\right)$. For computation of the magnetic field $\mathrm{H}_{\mathrm{z}}(\mathrm{x}, \mathrm{y}, \mathrm{z})$ the Biot-Savart law was also used.

According to formula (1), $\mathbf{d}$ is a distance from the point $M$ to the conductor element ds. Considering that $a_{1}$ is the distance from point $\mathrm{M}$ to the conductor PQ and $\beta$ is an angle between $a_{1}$ and $d$, we can write the final general formula for the magnetic field differential equation as follows:

$$
d H=\frac{I}{4 \pi} \frac{\operatorname{Cos} \beta}{a_{1}} d \beta .
$$

For the distances $\mathrm{a}_{1}, \overline{\mathrm{MP}}$ and $\overline{\mathrm{MQ}}$ we can write:

$$
\begin{gathered}
a_{1}=\sqrt{(p-z)^{2}+(q-y)^{2}} \\
\overline{M P}=\sqrt{(-L-x)^{2}+(q-y)^{2}+(p-z)^{2}} \\
\overline{M Q}=\sqrt{(L-x)^{2}+(q-y)^{2}+(p-z)^{2}} .
\end{gathered}
$$

After mathematical arrangements of computing of all four conductors and summing the particular field components

$$
\mathrm{H}_{\mathrm{z} 1}+\mathrm{H}_{\mathrm{z} 2}+\mathrm{H}_{\mathrm{z} 3}+\mathrm{H}_{\mathrm{z} 4}=\mathrm{H}_{\mathrm{z}} \text {, }
$$

the resultant formula has the following form

$$
\mathrm{H}_{\mathrm{z}}(\mathrm{x}, \mathrm{y}, \mathrm{z})=\frac{\mathrm{I}}{\pi} \sum_{\mathrm{n}=1}^{4} \sum_{\mathrm{i}=1}^{4} \mathrm{~W}_{\mathrm{in}} \mathrm{V}_{\mathrm{in}}
$$

where

$$
\mathrm{V}_{\text {in }}=\frac{\mathrm{q}_{\mathrm{i}}-\mathrm{y}}{\left(\mathrm{p}_{\mathrm{n}}-\mathrm{z}\right)^{2}+\left(\mathrm{q}_{\mathrm{i}}-\mathrm{y}\right)^{2}}+\frac{\mathrm{q}_{\mathrm{i}}+\mathrm{y}}{\left(\mathrm{p}_{\mathrm{n}}-\mathrm{z}\right)^{2}+\left(\mathrm{q}_{\mathrm{i}}+\mathrm{y}\right)^{2}}
$$

$\mathrm{W}_{\mathrm{in}}=\operatorname{Sin}\left[\operatorname{ArcTan}\left(\frac{\mathrm{L}-\mathrm{x}}{\mathrm{p}_{\mathrm{n}}-\mathrm{z}}\right)\right]+\operatorname{Sin}\left[\operatorname{ArcTan}\left(\frac{\mathrm{L}+\mathrm{x}}{\mathrm{p}_{\mathrm{n}}-\mathrm{z}}\right)\right]$

where

$n$ - index determining a numerical order of a wire in a quadrant,

$p_{n}$ - position of a conductor on the $\mathrm{z}$-axis for $\mathrm{n}=1$ to 2 both for horizontal and vertical conductors,

$q_{i}$ - half length of the vertical conductors,

$i$ - number of conductors,

$L$ - half length of the horizontal conductors.

By reciprocally exchanging the variables $\mathrm{x}$ and $\mathrm{y}$ we obtain an adequate expression both for horizontal and vertical conductors. The resultant magnetic field is a sum of four components according to formula (8). The current I of both horizontal and vertical conductors flows in anticlockwise direction.

For the phantom practical use it is necessary to construct a coil equipped with more wires, more turns. Computation results of a single rectangular coil were interpreted with the 3D-plot presentation of the magnetic field $\mathrm{H}_{\mathrm{z}}(\mathrm{x}, \mathrm{y}, \mathrm{z})$.

Computation results, 3D-plot of the magnetic field $\mathrm{H}_{\mathrm{z}}$ $(\mathrm{x}, \mathrm{y}, \mathrm{z})$ of the rectangular coil according to Fig.3. are depicted in Fig.4. The relative values for computation are as follows: $\mathrm{z}=1.8 ; \mathrm{p}=2 ; \mathrm{q}=4 ; \mathrm{L}=4 ; \mathrm{I}=100$;

For a simple example, a concentric rectangular four-coil system was designed. 


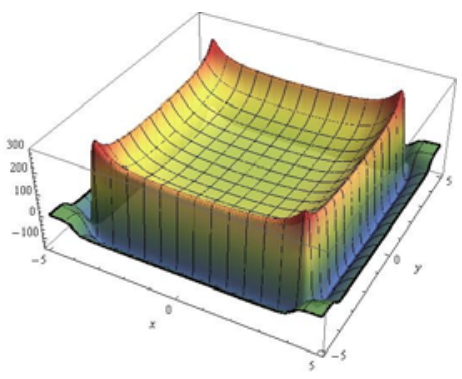

a)

Fig.4. Computation result - magnetic field $\mathrm{Hz}(\mathrm{x}, \mathrm{y}, \mathrm{z})$,

a) 3D Plot of the simple rectangular coil according to Fig.3.

b) Density-plot of relative values of the magnetic field $\mathrm{Hz}(\mathrm{x}, \mathrm{y}, \mathrm{z})$.

Following Fig.3, next relative parameters were chosen: $\mathrm{I}=100 ; \mathrm{p}=2 ; \mathrm{z}=1.8$; and relative dimensions of the four rectangular coils: $\mathrm{q}_{1}=1 ; \mathrm{L}_{1}=1 ; \quad \mathrm{q}_{2}=2 ; \mathrm{L}_{2}=2 ; \quad \mathrm{q}_{3}=3$; $\mathrm{L}_{3}=3 ; \mathrm{q}_{4}=4 ; \mathrm{L}_{4}=4$

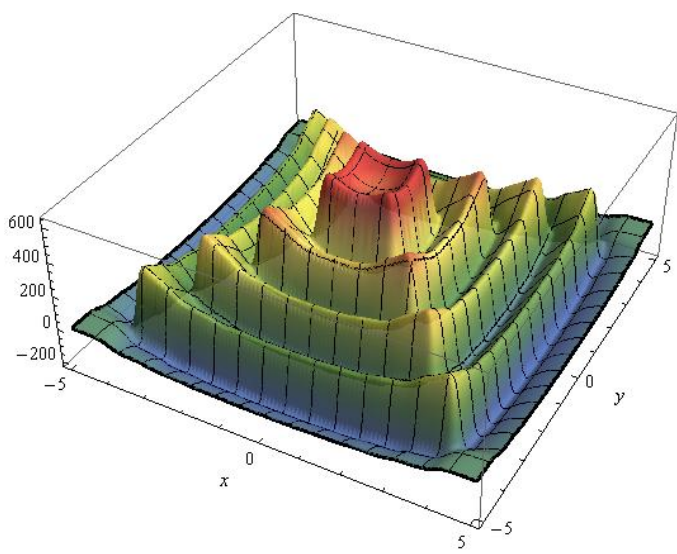

Fig.5. 3D-plot of relative values of the magnetic field $\mathrm{H}_{\mathrm{z}}(\mathrm{x}, \mathrm{y}, \mathrm{z})$ of the concentric rectangular four-coil system.

Computation results in the form of the 3D-plot of the magnetic field $\mathrm{H}_{\mathrm{z}}(\mathrm{x}, \mathrm{y}, \mathrm{z})$ of concentric rectangular four-coil system are depicted in Fig.5.

Using this phantom for 2-D NMR imaging a density-plot presentation of the magnetic field distribution is more suitable, see Fig.6.

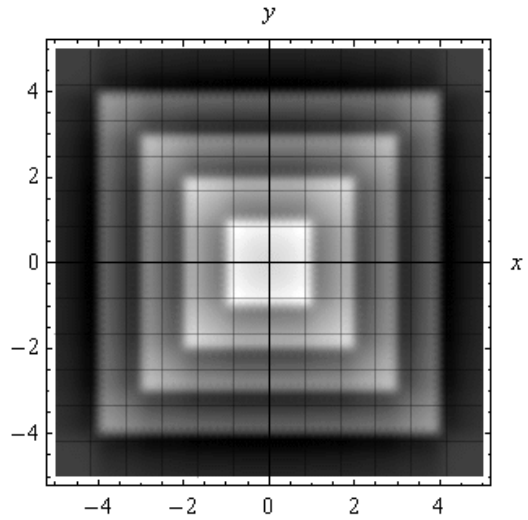

Fig.6. Density-plot of relative values of the magnetic field $\mathrm{Hz}(\mathrm{x}, \mathrm{y}, \mathrm{z})$ of the concentric rectangular four-coil system.

\section{EXPERIMENTAL RESULTS}

For our experiments an NMR imager (ESAOTE Opera), permanent magnet $0.178 \mathrm{~T}$ with vertical orientation of the basic magnetic field $\mathrm{B}_{0}$ was used. The phantom coil was placed between two plastic bushings filled with $0.1 \mathrm{wt} \%$ solution of $\mathrm{CuSO}_{4}$ in distilled water. $\mathrm{CuSO}_{4}$ was used to shorten the repetition time TR to $200 \mathrm{~ms}$ due to its reduction of $\mathrm{H}_{2} \mathrm{O} \mathrm{T}_{1}$ (for speeding up the data collection). The general configuration is shown in Fig.7. and Fig.8.

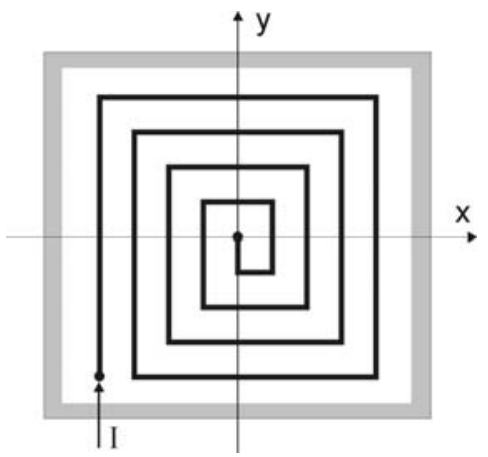

Fig.7. Meander planar coil design, dimensions 60 × $60 \mathrm{~mm}$, realized on a printed board.

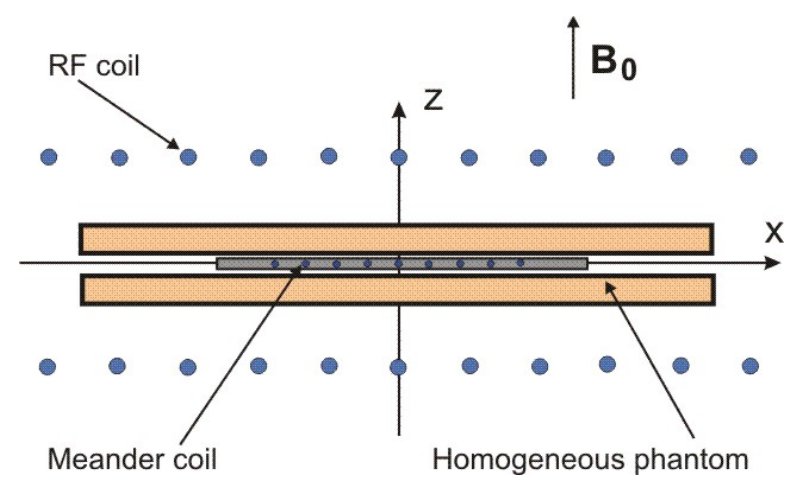

Fig.8. RF coil, active measuring volume (homogeneous phantom) and meander planar coil.

An RF transducing coil (solenoid) together with the phantom was placed into the centre of the electromagnet, perpendicular to the magnetic field $\left(\mathrm{B}_{0}\right)$ orientation [11]. Feeding current $\mathrm{I}=30 \mathrm{~mA}$ was applied to the meander coil creating a planar source of a weak magnetic field in the shape of a meander.

The gradient-echo NMR sequence (Fig.9) was selected for the measurement [12]. A special feature of the sequence is its sensitivity to basic magnetic field inhomogeneities.

A 5-lobe sinc pulse selectively excited the sample. The dimensions of the vessel with sample determined the slice thickness, therefore the slice gradient was switched off. Images were obtained with a field of view of $120 \mathrm{~mm}$. The number of samples and the number of views determining the final resolution was 128 each and the echo time TE was 18 ms. In order to increase the signal-to-noise ratio of the data, the signals were averaged 16 times. For magnetic field deformation representation a phase image was calculated using the phase unwrapping methods [4] and [13]. 


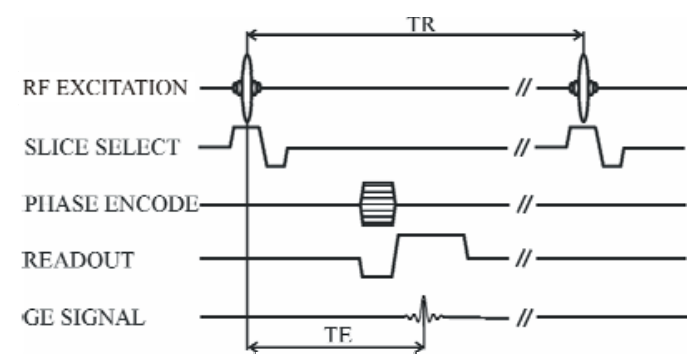

Fig.9. Time diagram of the NMR sequence for the gradient-echo signal detection. TE - echo time of $18 \mathrm{~ms}$, TR - repetition time of $300 \mathrm{~ms}$.

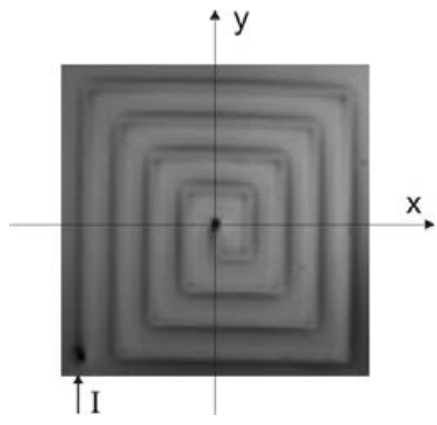

Fig.10. NMR image of the magnetic field distribution of a meander planar coil $(60 \times 60 \mathrm{~mm})$, number of voxels $150 \times 150$

An NMR image of the magnetic field distribution of a meander planar coil $(60 \times 60 \mathrm{~mm})$ measured by using gradient-echo imaging sequence is depicted in Fig.10.

The experiment revealed the following facts: It is evident that imaging of the magnetic field of the electromagnetic phantom can be performed only if the vector of the static magnetic field $\mathrm{B}_{0}=\mathrm{B}_{\mathrm{z}}$ is perpendicular to the phantom plain. Other orientations caused significant blurring of the image edges in the $\mathrm{x}$ - and $\mathrm{y}$-axis directions due to strong basic magnetic field $\mathrm{B}_{0}$.

\section{CONCLUSIONS}

A new method for mapping and imaging of the magnetic field of planar electromagnetic coil phantoms placed into the homogenous magnetic field of an NMR imager was proposed. The method is based on a projection of magnetic field of the electromagnetic coil into a homogeneous planar phantom and subsequent NMR imaging using a gradientecho sequence. The method was tested using a single planar coil - meander fed by electric currents and generating weak magnetic field. The aim of the paper was imaging of the magnetic field and using it for design and verification of basic types of planar electromagnetic phantoms.

Electromagnetic phantoms have become an additional tool for measurement, imaging and quality testing using NMR imaging methods. The advantages of the electromagnetic phantoms are:

- possibility to continuously change the magnetic parameters of the phantom,

- use as a standard for comparison of NMR imagers equipped with different magnetic fields,

- high stability of magnetic parameters,
- universality, repeatability.

As to some disadvantages we could speak about a need to have a controllable current power supply with high stability, necessity to have a homogeneous holder filled with solution of $\mathrm{CuSO}_{4}$ in distilled water and use of a very homogeneous printing board as a base of the planar coil.

The electromagnetic active phantoms are suitable for $\mathrm{S} / \mathrm{N}$ ratio testing and very useful for imaging pulse sequences adjusting and examination.

The first results showed the feasibility of the method and some of the possibilities offered in this field of research. The electromagnetic phantoms can serve as additional quality testing tools alongside geometrical and chemical phantoms generally used in NMR imaging systems.

\section{ACKNOWLEDGMENT}

The financial support by the Grant Agency of the Slovak Academy of Sciences, project no. VEGA 2/0142/08 and State program ŠPVV no. 2003 SP200280203 is gratefully acknowledged.

\section{REFERENCES}

[1] Joy, M., Scott, G., Henkelman, M. (1989). In vivo imaging of applied electric currents by magnetic resonance imaging. Magnetic Resonance Imaging, 7, 89-94.

[2] Callaghan, P.T., Stepisnik, J. (1995). Spatially-distributed pulsed gradient spin echo NMR using single-wire proximity. Physical Review Letters, 75 (24), 4532-4535.

[3] Sekino, M., Matsumoto, T., Yamaguchi, K., Irrigate, N., Ueno, S. (2004). A method for NMR imaging of a magnetic field generated by electric current. IEEE Trans. on Magnetics, 40, 2188-2190.

[4] Frollo, I., Andris, P., Přibil, J., Juráš, V. (2007). Indirect susceptibility mapping of thin-layer samples using nuclear magnetic resonance imaging. IEEE Trans. on Magnetics, 43 (8), 3363-3367.

[5] Bhagwandien, R., Moerland, M., Bakker, C., Beersma, R., Lagendijk, J. (1992). Numerical analysis of the magnetic field for arbitrary magnetic susceptibility distributions in 2D. Magnetic Resonance Imaging, 10 (2), 299-313.

[6] Willcott, M.R., Mee, G.L., Chesick, J.P. (1987). Magnetic field mapping in NMR imaging. Magnetic Resonance Imaging, 5 (4), 301-306.

[7] Tomasi, D., Panepucci, H. (1999). Magnetic fields mapping with the phase reference method. Magnetic Resonance Imaging, 17 (1), 157-160.

[8] Yang, Q.X., Wang, J., Collins, C.M., Smith, M.B., Zhang, X., Ugurbil, K., Chen, W. (2004). Phantom design method for high-field MRI human systems. Magnetic Resonance in Medicine, 52 (5), 1016-1020.

[9] Liu, F., Crozier, S. (2004). Electromagnetic fields inside a lossy, multilayered spherical head phantom excited by MRI coils: models and methods. Physics in Medicine and Biology, 49, 1835-1851.

[10] Kraus, J.D., Fleisch, D.A. (1998). Electromagnetics with Applications, 5th Edition. McGraw-Hill.

[11] Přibil, J., Frollo, I. (2007). Simple method of distributed tuning of RF sensor for NMR imaging system. Measurement Science Review, 7 (2), 25-29.

[12] Liang, Z.P., Lauterbur, P.C. (1999). Principles of Magnetic Resonance Imaging: A Signal Processing Perspective. New York: Wiley-IEEE Press.

[13] Andris, P., Frollo, I. (2009). Simple and accurate unwrapping phase of MR data. Measurement, 42, 737-741. 